

\title{
ASPECTS DE LA SYNTAXE ET DE SON ÉVOLUTION DANS LES VOYAGES AUX INDES ORIENTALES DE FRANÇOIS PYRARD DE LA VAL $(1611,1679)$
}

\author{
Daniel DROIXHE \\ Universités de Bruxelles et de Liège
}

Dans sa Biographie liégeoise de 1837, le comte de Becdelièvre consacrait une longue notice à François Pyrard de La Val, « célèbre voyageur, dont le véritable nom était Pyrau ». La Biographie universelle Michaud fait naître Pyrard à Laval, dans la Mayenne, à une date indéterminée ${ }^{1}$. Becdelièvre récuse fermement cette tradition. Pyrard « naquit au village de Stembert près de la ville de Verviers, au Marquisat de Franchimont, dans l'ancien pays de Liège, vers l'an $1570 »$.

Le biographe s'appuie sur le témoignage du fameux abbé Duval-Pyrau, autrement dit Henri Pyrard (Verviers, 1737-1800), lecteur du roi de Prusse dans les années 1780, auteur du Catéchisme de l'homme social et d'Agiatis (Cf. Mat, 1979). Accusé d'espionnage sous la Révolution, Duval-Pyrau se présenta dans sa défense comme le petit-neveu d'un homme qui avait bien servi la France, en quittant sa patrie pour chercher fortune à Saint-Malo où il devint le «premier armateur qui, sous la protection du gouvernement, fit voile vers les Iles Maldives ». Duval-Pyrau allègue lui-même des «archives» de son ancêtre pour authentifier la filiation et Becdelièvre la confirme par l'existence de « quelques papiers » détenus par une nièce de Duval-Pyrau, « qui lèvent tous les doutes sur la naissance de notre célèbre voyageur liégeois ».

Quelle que soit l'origine de celui-ci, son ouvrage offre aujourd'hui à l'historien et à l'ethnologue des informations dont la valeur a été soulignée par G. Bouchon, qui salue chez Pyrard, à propos de sa description de Goa

$<\ldots>$ les jugements d'un observateur exceptionnel ; une capacité à saisir un climat politique et social dans lequel des transformations futures sont en train de voir le jour ; le talent de donner vie à tout ce dont il traite (hôpitaux et prisons,

1 N. Vanwelkenhuyzen me communique que Pyrard n'apparaît pas dans : H. Pleticka, Die Entdeckung der Welt : ein Lexicon, Vienne, Ueberreuter, 1993 ; Fl. D'souza, Quand la France decouvrit l'Inde, Paris, L'Harmattan, 1995. L'Enzyklopadie der Entdecker und Erforscher der Erde de D. Henze (Graz, Akademische Druck- und Verlagsanstalt, Bd. 4, 1995) s'arrête avant le nom de Pyrard. Bouchon (1989 : 305) écrit que « Belges et Français discutent à propos de ses origines, mais il paraît vraisemblable qu'il est né dans le nord-ouest de la France, plus précisément à Laval, ou dans les environs ». 
coutumes, maisons avec fenêtres en nacre, techniques, marchés et célébration locales; visites à travers la ville, si précises que l'on peut localiser de monuments aujourd'hui disparus) ; à quoi s'ajoute un sens critique aigu, habil à montrer les différentes facettes de chaque chose.

(Bouchon, 1989:311)

Pyrard embarqua en mai 1601, à Saint-Malo, sur le Corbin, chargé par de marchands de trouver, en compagnie du navire le Croissant, un chemin pour le Indes. Après avoir relâché à l'île d'Annobon, aujourd'hui Pagalu, dans le golfe de Guinée, à Madagascar et dans les Comores, le Corbin fit naufrage aux Maldives, où Pyrard fut retenu par les insulaires jusqu'au début de 1607. Sor adaptation à la langue et aux mœurs du pays, quelquefois jalousée par ses compagnons, lui attirèrent après bien des « afflictions et misères » les faveurs du roi, qui résidait à Malé. Il y développa un petit négoce qui lui donna quelque richesse (il ne lui manqua, dit-il, que de pouvoir exercer la religion chrétienne). En 1607, le roi du Bengale attaqua les Maldives et emmena Pyrard, qui gagna Calecut, ou Calicut, sur la côte de Malabar, d' où il fit route au début de 1608 vers Cochin, avec trois de ses camarades. Arrêté par les Portugais, il y fut emprisonné, tomba malade et fut envoyé à Goa, dont l'hôpital était célèbre. Rétabli, il servit dans les troupes portugaises, ce qui lui permit de voir d'autres régions des Indes. Il prétend même avoir visité les îles de l'archipel indonésien. Remis en prison, il obtint sa libération en 1610 et regagna enfin la France via l'Afrique et le Brésil.

Le récit de ces instructives tribulations offre aussi un intérêt particulier en matière de langue, dans la mesure où il se présente sous différentes versions séparées par le demi-siècle pendant lequel se forma principalement le français classique (une traduction anglaise fut aussi imprimée au XIX ${ }^{\mathrm{e}}$ siècle et une traduction portugaise en 1944). On se limitera ici aux éditions qu'on a pu voir en utilisant les ressources locales. La relation a paru pour la première fois en 1611 sous le titre de Discours du voyage des François aux Indes orientales, etc. ${ }^{2}$ D'autres éditions montrent, à un degré qu'il n'a pas été possible d'évaluer, l'intervention des lettrés parisiens ayant mis la main au texte. Le Discours attira en effet l'attention de Jérôme Bignon, qui s'était fait connaître - avant sa douzième année! - par la publication d'une Description de la Terre Sainte (1600). Avide de nouveaux détails, Bignon suscita par ses entretiens avec le voyageur une relation plus étendue, lesquels «matériaux », «soigneusement transcrits », furent communiqués au géographe et historien Pierre Bergeron (selon Eyries dans la Biographie Michaud). Bergeron « les mit en ordre » et dès 1611 parurent les Voyages des François aux Indes orientales, Maldives, Moluques, et au Brésil, depuis 1601 jusqu'en 161133. Ce volume n'a pas été vu. La

Bibl. centrale de la Ville de Liège, Fonds Capitaine, 7349.

L'ouvrage connut une réimpression, en deux volumes, en 1615. 
narration, note la Biographie Michaud, y « est beaucoup plus détaillée que dans le premier ouvrage». "Quelquefois les circonstances diffèrent un peu entre elles ; mais le fond est le même ».

On a comparé le Discours, qui avoisinait les 350 pages d'un petit in-huit, à l'édition donnée en 1679 par Pierre Duval (1618-83), géographe royal, neveu du fameux Nicolas Sanson. Cette édition, qui a pour titre Voyage de François Pyrard de La Val, contenant sa navigation aux Indes orientales, etc., compte près de 700 pages in-quarto ${ }^{4}$. C'est dire qu'elle diffère considérablement du premier texte. On y retrouve de nombreux passages du Discours, mais intégrés dans une relation totalement refondue charriant une infinité d'informations nouvelles.

L'exercice auquel on va se livrer risque d'être dans une certaine mesure faussé par une confusion des plans chronologiques, puisqu'en l'absence de l'édition corrigée de 1611, on ne sera jamais sûr que le texte de 1679 n' offre pas des traits apparus dans celle-ci. En outre, il ne peut être question d'attribuer à Pyrard ou à ses «correcteurs-éditeurs » telle habitude syntaxique. Tout ce qu'on sera en mesure d'avancer, c'est que divers usages imputables en 1611 à Pyrard n'étaient plus courants ou acceptés en 1679 - sans que nous sachions toujours si cette désuétude est absolument typique de la seconde moitié du siècle, ou si elle s'amorce dans une rédaction antérieure, plus proche de l'usage parisien. En tout état de cause, les deux versions paraissent souvent traduire l'évolution que décrivent les historiens de la syntaxe du XVII ${ }^{\mathrm{e}}$ siècle. D'autre part, il faut souligner que les observations suivantes résultent d'une lecture particulièrement «transversale », surtout fondée sur la comparaison d'un certain nombre de passages communs, dans les chapitres traitant de la première partie du voyage, des Maldives et des castes de la société indienne «à Malabar ». S'en tenant aux aspects suffisamment documentés, on s'est limité à la syntaxe du groupe nominal, du pronom et du verbe. On s'est fondé sur les ouvrages d'A. Haase, F. Brunot et G. Spillebout, auxquels a été jointe l'excellente petite synthèse d'A. SancierChâteau.

\section{Le groupe du nom}

\subsection{L'article}

\subsubsection{L'omission de l'article, spécialement avec même, autre, tout}

L'ancien usage, qui pratiquait avec une certaine liberté la suppression de l'article indéfini ou défini, montre l'un ou l'autre cas de régression, de l'édition de 1611

4 Bibl. centrale de la Ville de Liège, Fonds Capitaine, 7348. Sauf indication contraire, on se réfère à la première partie de cet ouvrage, qui en compte trois. 
à celle de $1679^{5}$. On y lit respectivement, au chapitre traitant des bijoux portés par les Malgaches qui habitent «l'isle de Sainct Laurens »:

(1a) $\quad<\ldots>$ leurs ornemens sont brasselets de cuivre, d'estain ou de fer, dont ils font fort grand estat $<\ldots>$

$(1611: 25)$

(1b) Leurs $<\ldots>$ ornemens sont des brasselets $<\ldots>$ (1679: 26)

(2a) Ce Roy [de l'île de Malé, dans les Maldives] porte $<\ldots>$ une robbe de cotton $<\ldots>$ avecques boutons d'or massif $<\ldots\rangle$

(1611: 63)

$<\ldots>$ avec des boutons $<\ldots>$

$(1679: 158)$

La tendance à exprimer l'article se manifeste aussi dans les tours impersonnels et constructions attributives, conformément à la recommandation de Vaugelas qui qualifiait de «vieillie » l'omission de l'article indéfini dans ce type de séquence (Spillebout, 1985 : 55). La passage suivant concerne les mœurs des Indous de Calicut.

(3a) C'est chose admirable que de leur constance en la conversation des femmes, car bien que les garçons et les filles soient pesle mesle aussi nuds les uns que les autres, il ne leur eschaperoit pas pourtant une parolle lascive $\langle\ldots\rangle$

(1611:143)

C'est une chose admirable $<\ldots>$

$(1679: 275)$

Pourtant, l'omission de l'indéfini, tant singulier que pluriel, s'observe encore en 1679, en particulier dans les cas classiques où le substantif est déterminé par même et autre (Haase, 1935: §53-54, 112-114). Le premier extrait suivant évoque la rencontre du Corbin et du Croissant avec un bâteau hollandais qui paraît vouloir les attaquer. Les Français se mettent en défense et disposent sur le bordage la toile matelassée devant les protéger des balles :

(4) $\quad<\ldots>$ par mesme moyen nous commençasmes tous ensemble à bastinguer les navires tout à l'entour $<\ldots>$

$(1679: 5)$

(5) Leur profession [des Brameny ou brahmanes] est diverse selon qu' ils se veulent addonner, car il y en a qui se mettent aux armes entre les Nayres [ou Naires, nobles], faisant mesmes choses et habillez comme eux <...>

$(1611: 134-35 ; 1679: 266)$

5 Haase $(1935: \S 28,50)$ : «L'emploi de l'article, facultatif en ancien et en moyen français, ne devient obligatoire qu'à la fin du XVIe siècle... »; Spillebout (1985: 52 sv). 
(6) En ce mois [de décembre] vous voyez les garçons et les filles [des Maldives] se caresser et faire l'amour volontiers plus qu'en autre saison.

(1679:103)

À propos de l'usage concernant les séquences avec même, on notera que l'omission devant pareil semble moins bien acceptée en 1679 qu'en $1611^{6}$.

(7a) Ils [les Nayres] ont pareilles ceremonies et superstitions que les Bramany <...> (1611: 140)

(7b) Ils ont des ceremonies et des superstitions semblables à celles des Bramany $\langle\ldots\rangle$

(1679:264)

L'omission du défini dans une séquence avec tout paraît également avoir moins bien résisté, dans le cours du siècle.

(8a) Le païs [de Calicut] est fertile de toutes choses nécessaires pour la vie <...> (1611: 132)

(8b) $\quad<\ldots>$ de toutes les choses $<\ldots>$

(1679: 264)

On peut même croire que l'ellipse après tous n'était plus immédiatement comprise en 1679. Le passage suivant de 1611 a donné lieu à un contresens : la réécriture suppose que les « rapineurs » de grand chemin étaient « tous étrangers ».

(9a) Ils sont toutesfois sur le pais rapineurs et voleurs, qui destrousseroient les passants, et les tueroient sans misericorde, si on n'y prenoit garde. Mais tous estrangers et mesme les Malabares Mahommetans qui vivent parmy eux, ont de coutume de prendre un Nayre d'escorte pour aller par terre jusques à la ville $\langle\ldots\rangle$

(1611:132)

(9b) Mais ils sont tous estrangers, et mesme les Malabares Mahometans qui vivent parmy eux, ont de coutume de prendre un Naire d'escorte $\langle\ldots\rangle$ $(1679: 273)$

\subsubsection{L'omission de l'article devant grand}

On note une persistance particulière de la suppression de l'indéfini devant grand, que le mot fasse partie de la séquence (un) grand nombre ou qu'il marque

6 Cf. Spillebout (1985:55) : Je puis te contenter par une même voie/Et payer ton secret en pareille monnaire (Molière, Mélicerte, 1666, 37-38) ; Avec semblables propos Céladon se reprenait (d'Urfé, Astrée, 1607, I). On rapprocherait volontiers des ellipses précédentes l'omission qui continue de s'observer dans le passage suivant : les François ayans négligé infinies belles occasions $(1611: 3 ; 1679: 2)$. 
l'étendue ${ }^{7}$. Pyrard évoque de manière particulièrement précise les rites de funérailles aux Maldives.

(10) Enfin les parens et amis accompagnent le corps, et grand nombre de personnes qui se trouvent sans qu'on les prie ny qu' on les advertisse $<\ldots>$ $(1679: 117)^{8}$

Les débuts de l'expédition ne manquent pas d'animation, annonciatrice, constate rétrospectivement l'auteur, d'autres difficultés :

(11) $\quad<\ldots>$ il y eut grande querelle entre nostre Capitaine et le premier Facteur ou Commis, qui en vindrent presque aux mains $\langle\ldots\rangle$ (1679: 11)

Pyrard décrit aussi avec force détails les habillements respectifs des trois castes formant la société indienne. La « seule marque » qui distingue les brahmanes, vêtus « d'une jaquette de toile de cotton avec un turban blanc en teste et des souliers rouges aux pieds », consiste dans « un cordon de trois filets de cotton qu'ils portent sur leur chair en écharpe ». Le passage montre que le même type d'omission devant grand tend là aussi à régresser : $<\ldots>$ et ne sçauroit l'on faire plus grand'injure à un Brameny que de luy rompre son cordon $\langle\ldots\rangle$

(1611: 134)

$<\ldots>$ une plus grande injure $<\ldots>$

$(1679: 265)$

\subsubsection{Divers}

Haase constate que l'omission de l'article défini, en dehors de la recherche stylistique d'un La Fontaine ou d'un Scarron, s'observe « très rarement » devant des noms concrets quand ceux-ci ne sont pas en quelque sorte « analogues aux noms propres », dans la mesure où ils désignent «les seuls représentants de l'espèce ». Il fournit ainsi des cas d'omission devant paradis, enfer et terre, ce dernier terme prenant éventuellement un sens très concret : Ils la mettent dans de grands paniers sous terre, sur laquelle ils allument du feu (Regnard, Voyage de Laponie). On rapprocherait cet exemple de ceux où l'article, défini ou partitif, était omis dans l'ancienne langue et encore quelquefois à l'âge classique devant des noms désignant la matière, l'espèce ou une réalité abstraite, même si

7 Cf. Spillebout (1985: 55) : Est-ce donc si grande chose? (Bossuet, 1648, Brièv. vie).

8 Voir également : à cause qu'il [le bâteau le Croissant] tenoit plus grand nombre de personnes $(1679: 22)$ de mesme les femmes ont grand nombre de maris (1679: 116); on fait aussi grand nombre de morceaux d'or ou d'argent (1679: 118); qui désirent grand nombre de prestres à leur sépulture (1697: 119); avec grand nombre de loges (1679: 158), etc. 
« l'expression du partitif est posée comme nécessaire dès le début du XVII ${ }^{e}$ siècle, et notamment par Maupas ${ }^{9}$.

$<\ldots>$ toutes les viandes de ce pays-là ne prennent pas si bien sel que font celles d'icy $\langle\ldots>$

(1679:28)

(14) Car outre l'exacte maniere de vivre selon leur religion ils [les brahmanes] ont cela de particulier, qu'ils ne mangent jamais chair ny poisson, ny autre chose qui ayt euë vie $<\ldots>$

$(1611: 133 ; 1769: 265)$

Le XVII ${ }^{\mathrm{e}}$ siècle pouvait également omettre l'article dans les formules superlatives du type les personnes (les) plus galantes ${ }^{10}$. Haase note au chapitre des substitutions entre article défini et indéfini que le superlatif relatif se construit parfois avec un indéfini ${ }^{11}$. On en rencontre un exemple apparenté, dans le passage consacré aux mœurs amoureuses des Maldives.

Les garçons vont chercher des plus belles et des plus odoriferantes fleurs, qu'ils façonnent et guirlandent fort gentiment, et les envoient aux filles $\langle\ldots\rangle$ (1679: 103)

\subsection{Les adjectifs indéfinis}

Le trait le plus marquant concerne l'indéfini chacun, qui continue de s'utiliser ici comme adjectif, alors que, selon Haase, cet emploi devient rare au XVII ${ }^{e}$ siècle sauf chez La Fontaine (Haase, 1935: § 47, 102 ; Spillebout, 1985 : 86 sv.). Spillebout précise que si «chacun et chaque sont employés concurremment » avant 1650, « dans la deuxième moitié du siècle chaque semble bien voir éliminé chacun dans l'emploi d'adjectif ».

(16) Outre les Naybes [chefs des treize provinces des Maldives, qu'on ne confondra pas avec les Nayres ou nobles de Calicut] il y a en chacune province ou Atolon un homme commis de la part du Roy, pour cueillir et lever ses droicts et revenuz $\langle\ldots\rangle$

$(1611: 40 ; 1679: 146)$

(17) En attendant le retour des Flamans je demeuray donc auprès du Roy [de Calicut] qui me fit bailler un logis avec un serviteur, et outre me faisoit distribuer chacun jour deux panants, qui sont deux pièces d'or $\langle\ldots\rangle$ $(1611: 129-30 ; 1679: 261)$

9 Sansier-Château (1993: 24), qui signale qu' « on trouve encore des expressions comme gagner temps, donner réputation ». Haase $(1935: \S 28,51)$ enregistre une majorité d'exemples d'omissions concernant les termes matin, jour, nord, en dehors de ceux fournis par La Fontaine. Voir aussi Spillebout (1985: 55) : Et que Dieu l'a pétri d'autre limon que moi (Boileau, Sat., 1666, V, 24).

10 Haase $(1935: \S 29,57)$ : «Cet emploi devient plus rare à la fin du XVII ${ }^{\mathrm{e}}$ siècle ».

11 Haase (1935: § 57, Rem. III, 124) : «C'est une chose la plus aisée du monde » (Molière, Av., III, 1). 
(18) Il y a trois portes [dans les temples mahométans des Maldives, ou Mesquites], et à l'entrée de chacune porte par dehors, il y a un puits large, où l'on descend par des degrez <...>

(1679: 92)

\section{Les pronoms}

\subsection{L'expression du pronom personnel sujet}

\subsubsection{L'expression du pronom de la première personne}

Sauf quelques cas particuliers, l'expression du pronom personnel sujet est «perçue comme obligatoire dès les premières années du XVII ${ }^{c}$ siècle », alors que l'omission était courante à l'âge précédent (Ronsard, qui en recommandait l'expression, l'omet lui-même) ${ }^{12}$. Celle du pronom de la première personne, qu'on trouve encore chez Molière, Racine et surtout La Fontaine, suggérant l'expression relâchée ou marotique, s'observe dans le texte de 1611, mais semble disparaître dans celui de 1679. Dans le premier exemple cité, l'éditeur a rétabli le pronom ; dans les autres, le passage de 1611 comportant le trait a été supprimé.

(19a) Il y a beaucoup d'entr'eux [des nobles indous] qui s'adonnent, avec tous ces exercices et leur profession ordinaire des armes, à l'estude des sciences, et en ay cogneu plusieurs qui estoient fort sçavans aux Mathematiques, principalement en l'Astrologie <...> (1611: 138-39)

(19b) <... et j'en ay connu $<\ldots>$ (1679, 273)

Le cap de Bonne-Espérance une fois franchi, on découvre une erreur du pilote, sur la question de savoir où le convoi se trouve par rapport à Madagascar :

(20) Dequoy nostre General fut fort faché, et en colere contre le Pilote qui estoit Flamant, qui disoit avoir esté trois fois aux Indes : Et toutefois, il n'y cognoissoit que peu de choses : Et croy que les Flamans nous l'avoient baillé en intention de nous faire perdre $\langle\ldots\rangle$ (1611:16-17).

(21) L'isle de Sainct Laurens [Madagascar] est infiniment grande, et croy que c'est la plus grand'isle du monde $<\ldots>$ (1611:22)

12 Brunot, 1966 : III, $2^{\mathrm{e}}$ part., 478 sv. ; Haase, 1935 : § 8, 13-17 ; Spillebout, 1985 : 143 sv. ; Sansier-Château, 1993 : 40. 
Intervenant ici dans une séquence commençant par et, l'omission peut être considérée comme relevant dans une certaine mesure de la syntaxe des propositions coordonnées (voir ci-dessous), bien que le groupe apparaisse en général détaché de ce qui précède. Spillebout range des exemples analogues sous la rubrique du « sujet zéro ». Dans ceux qu'on vient de citer, l'ellipse accompagne le verbe croire : on se souviendra que Maupas, dans sa Grammaire et syntaxe française $(1607,1625,1632)$, continue d'accepter les expressions du type Vous m'avez bien conseillé et vous croirai une autre fois, à la différence des autres auteurs du début du siècle ${ }^{13}$.

\subsubsection{L'expression du pronom impersonnel}

On sait que le XVII e siècle pratique plus couramment l'omission de l'impersonnel il (types actuels mieux vaut tard que jamais, reste à savoir, etc.). L'ellipse s'observe surtout devant falloir. Le passage suivant traite du mariage aux Maldives, où des envoyés du futur époux s'informent auprès de la promise.

Faut aussi qu'ils sçachent de combien elle veut estre dotee $<\ldots$. (1611:48)

Le même type d'ellipse cảractérise aussi le texte de 1611, lorsqu'il est question de l'escorte que les étrangers prennent pour atteindre la capitale du royaume de Calicut :

$<\ldots\rangle$ et faut prendre ceste garde par ce qu'autrement il seroit impossible de passer à sauveté entre ces Nayres, et n'y a aucun qui n'en uze de la sorte pour aller à seureté $<\ldots . .>$

(1611: 127)

On aura noté l'autre omission de pronom impersonnel que comporte le passage. L'ellipse dans la séquence et faut se maintient parfois en 1679. Pyrard décrit ici les rites funéraires des Maldives.

Ces six hommes et six femmes [qui lavent le corps du défunt et le portent en terre] sont officiers publics, et faut qu'ils soient receus pour gens de bien et sans reproche $\langle\ldots\rangle$

$(1679: 117)$

Mais la tendance à exprimer l'impersonnel se manifeste dès 1611 et s'affirme par la suite. Les extraits suivants mélangent de manière caractéristique omission et expression du sujet.

(25) Le 7. de Fevrier 1602, il se leva une si grande et impetueuse tourmente à la coste de la terre de Natal, où il nous fallut repasser, du vent de suroest, que l'un de

13 Spillebout, 1985 : 143 : Aussi est-il [le roi]bien raisonnable, et crois que ceux qui feront les fous s'en trouveront mal (Malherbe, Lettre, 2 août 1618) ; Sansier-Château, 1993 : 42. 
nos batteaux qui estoit ataché à la poupe, avecques un gros cable, fut incontinent tout remply d'eau, et alla à fonds, et nous fut impossible de le sauver <...> (1611: 17-18)

(26) Et si elle [la future épouse, aux Maldives] dit avoir esté mariée, et que son mary l'eust quittée, ou qu'il fust mort, faut qu'elle le preuve par trois tesmoins $<\ldots>$. Plus il faut qu'il y ait trois mois que la répudiation ait esté faicte, de peur qu'elle fust grosse d'enfant, et qu'elle ait eu trois fois ses fleurs $<\ldots>$ (1611:48)

L'aboutisssement de l'évolution est illustrée dans ce passage de 1679, qui a trait au «cordon de trois filets de cotton » qui distingue les brahmanes.

(27a) < . > et ne sauroit l'on faire plus grande injure à un Brameny que de luy rompre son cordon, et faut qu'il en prenne un autre avec pareille solennité $<\ldots>$ (1611: 134)

$<\ldots>$ et il faut qu'il en prenne $<\ldots>$

$(1679: 265)$

\subsection{L'omission du pronom personnel sujet en structures coordonnées}

«L'ancienne langue omettait très arbitrairement de répéter le pronom personnel sujet devant des verbes coordonnés employés à un mode personnel. La langue du $\mathrm{XVII}^{\mathrm{e}}$ siècle suit souvent cet usage » (Haase, 1935 : $\$ 147,400$ sv.). L'édition de 1611 en fournit d'innombrables exemples. Il est plus difficile de définir la situation de 1679, parce que d'un côté, elle répète le sujet dans des cas également nombreux tout en laissant subsister des omissions caractéristiques, quand les propositions coordonnées n'ont pas le même sujet et que le pronom personnel omis renvoie à un antécédent en position d'objet.

(28) A une lieuë et demie d'Anabon [Annobon] il y a une petite isle qui est si couverte d'oiseaux, que l'on ne sçauroit presque marcher en aucun endroict qu'on ne marche dessus, ou sur leurs œufs. On les nomme Pingui, et sont un peu plus gros que nos pigeons $\langle\ldots\rangle$

$(1679: 11)^{14}$

On comparera cet exemple au suivant.

(29a) Outre les Naybes il y a en chacune province ou Atolon un homme commis de la part du Roy, pour cueillir et lever ses droicts $\langle\ldots\rangle$. On les nomme Varvery, et sont fort respectez et honorez $<\ldots>$

(1611: 40)

14 L'omission est d'autant plus courante dans le cas de structures coordonnant ou reliant, même avec incise, des pronoms impersonnels : il nous conveint baisser les voilles, et l'un de nos Mariniers tomba en la Mer et se noya, et nous fut impossible de le sauver (1611: 16); Il tombe dans ceste baye une rivière de ladite isle, en laquelle y a grand nombre de crocodiles (1611:23). 
$(29 b)$

$<\ldots>$ et ils sont fort respectez $<\ldots>$

(1679: 146)

La tendance dominante est à la répétition du sujet, même quand celle-ci ne serait pas nécessaire dans le français d'aujourd'hui.

(30a) La France a négligé l' art de la marine : Nous en recognoissons à present mieux que jamais, et en ressentons les oncommoditez $<\ldots>$ $(1611: 3)$

(30b) $\quad<\ldots>$ et nous en ressentons $<\ldots>$ $(1679: 2)$

Un autre exemple, pris dans le chapitre décrivant l'existence des reines des Maldives :

(31a) Quand elles sont grosses elles sortent aussi pour aller se baigner en la mer; comme toutes les autres femmes : carc'est la coustume du païs, et tiennent que cela est fort sain $\langle\ldots\rangle$

(1611:67)

(31b) $\quad<\ldots>$ et elles tiennent $<\ldots>$ (1679: 146)

L'explicitation du sujet en strucures coordonnées n'est du reste que la manifestation d'une tendance plus générale. L'exemple suivant, où l'ellipse peut être influencée par la présence de même, montre aussi, dans les derniers mots, un souci de symétrie typiquement classique.

(32a) Et bien qu'il y ait plusieurs Provinces et diversité de contrées [sur la côte de Malabar] ils sont tous neantmoins d'une mesme langue, loy et religion, mesme police, ordre et distinction du peuple et des races $\langle\ldots\rangle$ (1611: 130)

(32b) $\quad<\ldots>$ ils sont tous neantmoins d'une mesme langue, d'une mesme loy et religion, d'une mesme police, mesme ordre et distinction de peuple et de races $<\ldots>$

$(1679: 263)$

\subsection{L'emploi pléonastique du pronom personnel sujet}

« Le pronom de la troisième personne rappelait dans l'ancienne langue beaucoup plus souvent qu'aujourd'hui des membres de phrases qui le précèdent ou qui le suivent » (Haase, 1935 : § 6, 8 sv. ; Spillebout, 1985 : 144-45 ; Sansier-Château, 1993 : 41). Dès le début du siècle, on tend à supprimer le pléonasme, qui reste néanmoins très usuel et subsiste dans le discours de réception de Boileau à l'Académie française. Plusieurs cas sont à distinguer. 
L'exemple suivant accumule deux des circonstances énoncées par Haase : le sujet se trouve séparé du verbe par des participes commandant des propositions relatives. On y décrit les démarches accomplies par le père d'un candidat au mariage, ou par un proche parent du côté paternel.

Celuy là avec le mary futur se presentant devant le Pandiare ou Naybe, lequel estant certifié de tout ce qu'il desire, prenant la main du marié qui est present, il luy demande s'il veut bien prendre la femme aux conditions qui ont esté auparavant proposées $\langle\ldots\rangle$

$(1679: 112)$

On conviendra que l'enchevêtrement des acteurs exigeait bien la répétition du sujet, sans que celle-ci allège vraiment la compréhension. Cet extrait illustre aussi le cas apparemment fréquent, distingué par Spillebout, où le pronom de rappel a pour antécédent un pronom démonstratif suivi d'une relative. En voici un autre exemple :

(34) Tellement que ceux des nostres qui estoient à bord du Croissant voyans que la mer s'enfloit, ils se mirent dans le gallion ou basteau et s'en retournerent vers nous $\langle\ldots\rangle$

$(1679: 16)$

Si la seconde édition accepte encore certains pléonasmes anaphoriques, la tendance pousse néanmoins à une réécriture qui les élimine. Ce passage raconte la rupture du mât de misaine du Corbin. On avertit le commandant de l'expédition, « nostre General »,

(35a) $<\ldots>$ lequel estant resolu de continuer nostre route, sans s'arrester à ceste occasion, il nous envoya les charpentiers de son navire $<\ldots>$ $(1611: 7)$

(35b) $\quad<\ldots>$ mais ayant resolu de continuer nôtre route, sans s'arrester à cette occasion, il nous envoya les charpentiers $<\ldots>$ (1679: 263)

(36a) A tous ceux qui arrivent [aux resjoüissances de mariage, chez les Indous], mesme aux estrangers on leur presente un plat de betel $\langle\ldots\rangle$ (1611: 154)

(36b) On presente à tous ceux qui arrivent $<\ldots>$ $(1679: 282)$

On n'a relevé qu'un exemple de pléonasme cataphorique. L'annonce du sujet, en l'occurrence une proposition introduite par un pronom relatif nominal, répond au canonique bel esprit il ne l'est pas qui veut des Femmes savantes. Le passage évoque la distribution, lors des funérailles, de morceaux d'or ou d'argent mis dans de petites pièces de toile. Le bénéfice en est théoriquement réservé aux brahmanes, mais

$<\ldots>$ il en prend qui veut, selon les moyens du defunct et de ses heritiers $<\ldots>$ (1679: 118) 


\subsection{Les pronoms démonstratifs}

\subsubsection{L'extinction du pronom neutre}

L'emploi du pronom ce était déjà, au XVI siècle, « considérablement restreint par l'apparition du pronom composé » (Haase, $1935: \S 18,36$ sv. ; Spillebout, 1985 : 70 sv. ; Sansier-Château, 1993 :53-54). On sait qu'il se réfugie au XVII ${ }^{e}$ dans la langue familière ou dans des locutions plus ou moins figées - acceptées par Vaugelas mais évocatrices du « style des notaires », d'où leur présence dans les Plaideurs. On le relève de temps en temps dans l'édition de 1611 du Voyage, en particulier dans l'expression du type ce dit-on. Nous retrouvons les distributions d'argent lors des funérailles aux Maldives.

(38) Mais ils [les assistants ayant prié pour le défunt] n'en prennent pas tous, dautant que cela n'apartient qu'aux gens d'Eglise, ce disent-ils $<\ldots .>$ (1611:53)

Toutefois, la tendance visant au remplacement de ce par ceci ou cela, ou à l'élimination du pronom, se marque nettement, mais sans surprise, dans le texte de 1679.

(39a) Le Jeudy au soir qui est la veille du jour de la feste, ils prient pour les trespassez, et pour ce ils preparent à manger et à boire qu'ils laissent aux prestres de leur loy $\langle\ldots\rangle$

$(1611: 42)$

(39b) $\quad<\ldots>$ et pour cela ils preparent $<\ldots>$ (1679: 96)

(40a) [Les étrangers doivent se faire escorter] moyennant ce on peut aller seurement par toute la contree de Malabar <...> (1611: 139-40)

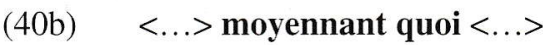
(1679: 273)

(41a) Toutesfois à cause de ce [la polygamie des Indoues] il y a incertitude de sang, et ne peut-on discerner au vray à qui sont les enfans $\langle\ldots\rangle$

(1611: 143)

(41b) Toutefois à cause de cela $\langle\ldots\rangle$

(1679: 275)

\subsubsection{Icelui, cettui-ci}

Icelui n'est plus employé au XVII ' siècle que par les juristes, les médecins ou pour des raisons stylistiques; Spillebout le rencontre aussi dans le premier tiers du 
siècle chez « des écrivains qui, par leur formation, se rattachent directement au $\mathrm{XVI} \mathrm{I}^{\mathrm{e}}$ siècle $»^{15}$. La forme apparaît encore à plusieurs reprises dans l'édition de 1679 .

(42) En l'une d'icelles, appelée l'isle de Mayo [une des îles du Cap-Vert], se trouve une si grande quantité de sel en rochers, que l'on en peut charger tant qu'on veut $\langle\ldots\rangle$

(1679 : 5 ; l'édition de 1611 offre un texte tout différent)

(43) On le nomme Cap des Aiguilles [à l'est du cap de Bonne-Espérance], parce qu'au droit d'iceluy les compas ou esguilles demeurent fixes, et regardent directement le Nort <...>

(1679: 14)

(44) les Naybes ou chefs de provinces sont prestres et docteurs de leur loy, qui ont l'oeil à tout ce qui est de la religion, et instruction du peuple en icelle $<\ldots>$

$(1611: 38 ; 1679: 144)$

On note encore fréquemment cettuy-ci chez un modèle comme Balzac. Malherbe l'emploie également, comme Théophile de Viau, mais il devient rare après Corneille. Il subsiste occasionnellement, une génération plus tard, dans le Voyage.

(45) Nous fusmes accompagnez de toutes sortes de malheurs durant le sejour que nous fismes en cette rade ; Car outre cettuy-ci il nous en arriva encore d'autres, particulierement en nostre navire $<\ldots>$ (1679: 11)

\subsubsection{L'omission du pronom démonstratif}

L'ancien français omettait volontiers le déterminatif ce devant une proposition relative « résumant une proposition antérieure » (c'est le « démonstratif zéro » de Spillebout - Haase, 1935 : $\$ 35,67-68$; Spillebout, $1985: 73$ sv.). Bien que l'expression du pronom neutre se généralise en moyen français, nos deux Voyages usent régulièrement de l'ancienne liberté, comme le fait aussi le XVII siècle :

(46) le mas de misene de nostre vaisseau se rompitet esclata, qui fut un commencement de malheur $<\ldots>$

$(1611: 7 ; 1679: 3)$

15 Haase, $1935: \S 23,43$ sv. ; Spillebout, $1985: 68-69$, dont le classement des faits parait ici moins clair que chez Haase. Cf. le wallon Sav' bin qui dj' fê, qwand i sont f'nous? (Savez-vous bien ce que je fais, quand ils sont venus ?) dans le premier des Dialogues de paysans (1631-1636) édités par J. Haust (Liège, VaillantCarmanne, 1939, v. 64). 
(47) [le célèbre hôpital de Goa a été] fondé par les Roys de Portugal de vingt mil Perdos, qui valent chacun 25. sols piece. Qui est la un grand revenu pour tel effet $<\ldots .>$

$(1611: 195 ; 1679: \text { II, 3-4) })^{16}$

L'époque continuait aussi d'autoriser l'omission du pronom déterminatif « de rappel » dans la construction avec de + substantif/infinitif(Haase, $1935: \S 27,49$ sv.). Le passage suivant, relatif aux Maldives, exprime bien une certaine hésitation de l'éditeur de 1679 puisque le pronom absent de la version précédente est explicité, tandis qu'une addition l'omet deux lignes plus bas, malgré ce qui sépare les deux membres de phrase.

(48a) La religion qu'ils tiennent [aux Maldives] est de Mahomet $\langle\ldots>$ (1611:41)

(48b) La religion qu'ils tiennent est celle de Mahomet, et il n'y en a point d'autres par toutes ces isles, si ce n'est [celle] des estrangers qui y abordent $\langle\ldots\rangle$ (1679: 92)

\subsection{Les pronoms relatifs}

On a évoqué plus haut le cas de qui employé pour ce qui. Un seul autre trait a été noté par ailleurs. Vaugelas a condamné la persistance de duquel, avantageusement remplacé par dont, «mot plus doux », et H. d’Urfé évite déjà la forme composée qui ne se maintiendra que comme formule juridique (Sansier-Château, 1993 : 60). Les Voyages l'emploient dans la première édition et la conservent occasionnellement dans la seconde.

(49) Il y a aussy grande quantité de poisson, duquel nous prenions autant que nous en voulions $\langle\ldots\rangle$

(1611:23)

(50) Et au contraire on void que les nations, desquelles le terrouër estoit maigre, sterile, ou fort estroit, ont voulu moyenner leur recompense par la navigation $<\ldots>$

$(1611: 2 ; 1679: 1)$

\subsection{Les pronoms indéfinis}

Chacun continue, ainsi qu'on l'a vu, de s'employer comme adjectif dans les Voyages de Pyrard, y compris dans l'édition de 1679, même si « la distinction

16 Voir encore : De fait ils passerent au dessous du vent, qui est la plus grande marque de soumission qu'on puisse faire en mer (1679:4); qui s'appelle Samory, qui est une marque singuliere de sa grandeur pardessus les autres (1679: 164). Ces deux passages n'ont pas d'équivalent dans l'édition de 1611. 
entre forme déterminative et forme pronominale est faite dès le début du XVII ${ }^{\circ}$ siècle » (Sansier-Château, 1993: 64 ; Spillebout, 1985 : 88 sv.). Par contre, l'emploi pronominal d'aucuns dans le sens de quelques-uns semble régresser (Spillebout écrit que la forme plurielle, comme adjectif ou pronom, reste « couramment utilisée au XVII e siècle avec valeur positive »). Le texte de 1679 l'évite en tout cas, dans un passage où il est question, pour Pyrard, de faire route jusqu'aux Indes en compagnie d'un navire hollandais.

(51a) Ce que nous ne peusmes, à cause qu'il leur failloit aller passer par entre la terre ferme et l'isle Sainct Laurens [Madagascar], ainsi que nous dit leur General, pour aller trouver aucuns de leurs compagnons $\langle\ldots\rangle$ (1611: 15)

(51b) $\quad<\ldots>$ pour trouver leurs compagnons $<\ldots>$ (1679:15)

L'emploi caractéristique du pronominal un pour quelqu'un demeure « très usité » au XVII ${ }^{e}$ siècle selon Haase, qui note cependant que Corneille, après l'avoir pratiqué, remplacera systématiquement la première forme par la seconde en 1660 (Haase, $1935:$ 49, 105). Le changement affecte les deux versions du Voyage. Pyrard y donne de précieux renseignements sur le système et les usages matrimoniaux des peuples qu'il rencontre. Il ne s'offusque guère de l'usage qui permet à chaque femme, chez les nobles de Malabar, de « prendre jusque à trois marys tout ensemble », alors que « les Nayres ne peuvent avoir qu' une femme en mesme temps ».

(52a) La commodité qu'ils retirent de ceste coustume est qu'un qui n'a pas moyen de nourrir une femme peut avoir le tiers d'une seulement, et ne luy couste à nourrir qu'à ceste proportion $\langle\ldots\rangle$

(1611: 143)

$$
<\ldots>\text { est, qu'un homme qui <...> }
$$

$(1679: 274-75)$

Mais l'ancienne habitude syntaxique subsiste parfois, sans qu'on puisse dire si l'éditeur de 1679 suit machinalement le texte antérieur ou s'il accepte encore la forme. Le passage suivant concerne la «superstition » qui prohibe, chez les Indous, le contact physique avec les étrangers ou personnes d'une autre religion. Pyrard l'a souvent expérimentée, «m'estant voulu asseoir aupres d'eux sans y penser $\gg$.

Ainsi j'ay veu que s'ils vouloient bailler quelque chose, comme un baston ou une espece à un de religion diverse ils le jettent en la main de l'autre, pour ne le toucher $\langle\ldots\rangle$

$(1611: 150 ; 1679: 279)$ 


\section{Le verbe}

\subsection{L'infinitif prépositionnel}

On traite ici des cas où l'infinitif ayant valeur de proposition circonstancielle a le même agent que celui de la proposition principale ou possède un sujet propre (Haase, 1935 : $§ 85$ C-D, 198-201 ; Spillebout, 1985 : 316 sv.). Le fait le plus marquant consiste dans le remplacement de cet infinitif par une proposition plus ou moins correspondante ou dans une refonte du texte permettant de l'éviter. La révision de 1679 manifeste donc là un souci de clarté dépassant ce qu'exigeait la syntaxe courante, dans la mesure où le XVII ${ }^{e}$ siècle déconseillait seulement comme équivoque, tout en l'employant couramment, le tour où le sujet des deux membres de phrase n'est pas identique. Le premier des extraits qui suivent, relatif au mode de vie des brahmanes, offre un exemple - le seul relevé - d'infinitif prépositionnel qu'introduit par, cas effectivement considéré comme « déjà rare au XVII ${ }^{\mathrm{e}}$ siècle ».

(54a) $\quad<\ldots>$ [ils] ne boivent que de l'eau, gardans ceste austerité de pere en fils et en toute leur race, par ne se mesler et ne s'allier avec autres sortes de personnes $\langle\ldots\rangle$

(1611: 133)

(54b) $\quad\langle\ldots>$ toute leur race ; [ils ont cela de particulier] qu'ils ne se meslent $<\ldots>$ $(1679: 265)$

(55a) Quant au Roy de Coilan pour estre eslongné et tout à la pointe du Cap Commorin, il se maintient plus souverainement que les autres $\langle\ldots\rangle$ (1611: 131)

(55b) Quant au Roy de Coilan, d'autant qu'il est éloigné <...> (1679: 264)

Ce type de correction s'applique aussi à des participes présents.

(56a) Encore qu'ils [les nobles de Malabar] soient toujours nourris aux armes, qu'ils soient fort vaillans et determinez, ne faisant conte de leur vie, ce neantmoins ce sont les personnes les plus douces et humaines en conversation qu'on sçauroit dire $<\ldots>$

(1611: 139)

(56b) $<\ldots>$ d'autant qu'ils ne font point de conte de leur vie <...> (1679: 273)

La réécriture de la phrase se comprend mieux encore quand l'agent de l'infinitif introduit par pour avec valeur causale est particulier, la construction qui en résulte montrant dès lors une certaine lourdeur, même si elle garde dans le texte de 1611 un charmant parfum d'archaïsme. 
(57a) De sorte que le 27. de Decembre sur le minuyict, nous nous trouvasmes fort pres de terre, et n'eust esté un Marinier qui l'apperceut d'aventure, nous nous fussions perdus, pour estre la Mer en ces lieux là fort orageuse <...> (1611:13-14)

(57b) $\quad<\ldots>$ nous nous fussions perdus : car la mer estoit fort grosse et orageuse $\langle\ldots>$

$(1679: 14)$

\subsection{La construction de l'infinitif régime}

Les deux versions mettent particulièrement bien en évidence l'évolution qui affecte la syntaxe des infinitifs régis par des verbes exprimant l'obligation, sous les aspects du commandement ou de la contrainte («empeschement et destourbier », comme dit Maupas en 1632), ainsi que par des verbes de sentiment (Brunot, 1966 : III, $2^{\mathrm{e}}$ partie, 551 sv. et IV, $2^{\mathrm{e}}$ partie, 968-76 ; Sansier-Château, 1993 : 74-75). Alors que la construction est directe en 1611, comme pour les verbes « exprimant la pensée et la volonté », le texte de 1679 introduit systématiquement la construction avec $d e$.

(58a) $<\ldots>$ après avoir navigé deux jours et deux nuicts ne la pouvant voir [la terre], il [le « général » de l'expédition] commanda mettre le cap en l'autre bande <...> (1611:16)

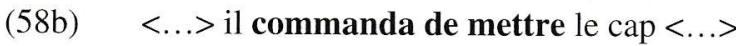
(1679: 16)

(59a) Ils [les prêtres des Maldives] vivent de certaine partie de fruicts que chacun est tenu leur bailler $<\ldots>$

$(1611: 38)$

(59b) < . > est tenu de leur bailler $<\ldots>$ $(1679: 44)$

Le changement caractérise particulièrement la syntaxe de contraindre. Si Malherbe, comme le note F. Brunot (1966 : 553), blâmait la construction directe, Maupas l'acceptait en même temps que les deux autres constructions possibles, c'est-à-dire avec à ou de. Les époux des Maldives peuvent se quitter «d'un commun consentement » :

(60a) Car si la femme ne le vouloit, l'homme seroit contrainct luy payer son doüaire $<\ldots>$

(1611 : 50)

(60b) $\quad<\ldots>$ de luy payer $<\ldots>$

(1679: 114) 
Le passage suivant montre néanmoins que commander pouvait se construire avec de dans le texte de 1611 - en même temps qu'il illustre la construction directe après un verbe de sentiment, également corrigée par la suite. L'introduction de la préposition de caractérise particulièrement le régime de craindre $^{17}$.

(61a) Incontinent nostre General commanda de resortir du dedans de ladite isle, et de revenir par la bande de dehors, pour ce qu'il craignoit ne pouvoir passer à cause des vents $\langle\ldots\rangle$

$(1611: 17)$

$<\ldots>$ de ne pouvoir pas passer $<\ldots>$

$(1679: 16)$

\subsection{Les formes en -ant}

Les deux extraits suivants illustrent d'une part l'indifférence du français classique à notre règle voulant que le participe présent ou le gérondif aient le même agent que le sujet du verbe principal, et d'autre part l'accord du participe présent avec un agent masculin pluriel (Haase, 1935 : § 91, 209 sv. ; Spillebout, 1985 : 231).

On détachera pour sa portée morale, parmi bien d'autres exemples, cette observation concernant l'hindouisme ${ }^{18}$.

Ils connoissent bien qu'il y a un Dieu, mais ils disent qu'estant bon, il ne le faut point prier ny l'adorer, puisqu'il ne fait point de mal $\langle\ldots\rangle$

$(1679: 279)$

Une autre coutume de ces régions consiste, chez les nobles, à percer « le gras de l'oreille aux jeunes enfans, tant masles que femelles », et à remplir « le vuide de petits rouleaux de feuilles de palmites »:

17 Cf. Spillebout, 1985 : 261 : Grandmercy, je craindrois vous donner trop d'ennuis (Esternod, Espadon Sat., IV, 1619, 49); Je ne crains point faillir, quoy que ma Muse die (Th. de Viau, Sat. 2de, 1621, 50). Brunot (1966:554 et 974) écrit qu' on trouve encore, au XVII siècle, « de rares exemples » de la construction sans préposition, que craindre à, non formellement condamné par Malherbe, s'avère assez fréquent et que les constructions avec à et de sont admises de Maupas à Bouhours, la seconde étant recommandée par Oudin.

18 Ainsi : Estant lavé ils le couvrent [le corps du défunt] et le garnissent de coton $(1679: 116)$; Estant presenté au Roy, il me feist enquerir par sontruchement en langue Portuguaise d'où j'estois (1611:128); Enfin m'ayant demandé commej'estois parvenu à Calecut et à quel dessein, et luy ayant conté tout ce qui m'estoit arrivé, et que jen'estois venu qu'en intention d'y trouver les Hollandois, que l'on m'avoit dit estre les bienvenus, il me dit lors qu'à la verité, etc. (1679:260). On note à ce propos que Pyrard, « bien qu'indigné par les rigueurs de l'Inquisition et la suspicion que fait régner le Saint Office, met aussi en évidence les actions des religieux en faveur de la tolérance » (Bouchon, 1989 : 312). Emprisonné à Cochin, il doit sa libération à un jésuite ; il trouve de l'aide auprès des Pères qui enseignent à Goa, dont le fameux Thomas Stephens ou Stevens, cité dans les histoires de la linguistique pour avoir observé l'allure occidentale de certains mots sanskrits. 
(63) Estimants une grande beauté d'avoir les oreilles ainsi grandes, qu'ils remplissent par apres d'or et de pierreries $\langle\ldots\rangle$

(1611: 142)

\section{Conclusion}

Il y a au moins deux manières d'interpréter les résultats ci-dessus. Celles-ci sont de tout façon limitées par l'absence de prise en compte de l'édition revue de 1611, qui permettrait de dire dans quelle mesure l'éditeur de 1679 a appliqué de nouveaux usages syntaxiques, par rapport à l'originale (en l'absence d'un éventuel manuscrit laissé par Pyrard). Il faudrait aussi faire intervenir une différence possible de traitement, selon les parties ou passages qui ont été repris et réécrits : on note pour certains (notamment dans la seconde partie de la version étendue) une reproduction plus mécanique du Discours de 1611, qu'on imputerait volontiers à la lassitude.

On peut d'abord considérer que les différences relevées dans cette édition de 1679 traduisent exactement les changements intervenus dans le cours du siècle, tels que décrits dans les travaux utilisés. Cette part de l'inventaire est sans surprise : les corrections de 1679 illustrent la tendance à exprimer l'article indéfini sauf devant même, autre ou grand, l'obligation d'exprimer le sujet à la première personne, avec tolérance devant croire, la répétition du pronom sujet en structures coordonnées, la réduction des pléonasmes anaphoriques du même pronom, l'extinction du neutre ce en fonction d'objet ou de complément, etc.

La comparaison avec la chronologie et typologie générales que définissent les histoires de la syntaxe dégage quelques traits particuliers. L'omission de l'article défini devant un substantif accompagné de tout paraît moins bien acceptée à mesure qu'on avance dans le siècle, par rapport à l'ellipse devant même et autre. Le défini s'omet parfois tardivement devant des noms de matière. Chacun continue à l'occasion de s'employer comme adjectif dans la version de 1679 , alors que chaque est censé l'avoir éliminé - mais on paraît éviter l'ancien emploi d'aucuns, à un moment où il serait encore toléré. L'obsolète un pour quelqu'un ne disparaît pas complètement. Globalement, la réécriture montre plutôt la persistance d'usages anciens, voire d'archaïsmes. Si elle tend à exprimer le pronom impersonnel devant falloir, elle maintient aussi l'omission, quelquefois. L'incertitude règne, y compris à partir du début du siècle : le texte de 1611 peut adopter dans un même passage deux attitudes différentes en matière d'expression ou d'omission du sujet impersonnel. Une hésitation résiduelle s'observe également en ce qui concerne l'expression du sujet en structures coordonnées et celle du pronom démonstratif « de rappel » en construction déterminative. On accepte encore l'ellipse du premier, en 1679, y compris quand le sujet omis ne renvoie pas à celui de la proposition coordonnée. On trouve aussi dans un même passage la 
correction de l'ancien tour leur religion est de Mahomet, qui devient leur religion est celle de Mahomet, et une nouvelle ellipse quand il est question de (celle) des étrangers. Mais le souci d'explicitation, répétons-le, est largement dominant, ainsi que la tendance visant au remplacement des infinitifs prépositionnels ou des participes présents par des propositions. Le français classique réclame en même temps simplification analytique de la lecture et séduction rythmique de la phrase, deux aspects, à tout prendre, d'une même démarche de persuasion.

Une seconde manière d'envisager les résultats ferait valoir que l'éditeur de 1679 a maintenu pour des raisons stylistiques divers archaïsmes. Utiliser icelle ou cettuy-ci, faire traverser par l'imagination chacune province de Madagascar ou les nations desquelles le terrouër estoit maigre conservait au texte son authenticité de récit pionnier. La rudesse de certains tours rendait sensibles les difficultés et l'audace de l'expédition. En même temps, la simplicité un peu vieille d'autres formules enlumine la découverte émerveillée des pays traversés, décrits de manière souvent touchante, par les accents vécus, et avec un souci du détail qui justifierait que l'œuvre soit davantage étudiée en tant que document ethnographique. Commel'écrit G. Bouchon, « the time has come indeed to reread Pyrard in the light of recently accomplished work on these fields ».

\section{Références bibliographiques}

Becdelièvre (1837), comte de, Biographie liégeoise, Liège, Jeunehomme.

Bouchon, G. (1989), A French traveller in Portuguese India (1601-1610) : François Pyrard de Laval, Studia, 49, 301-14 (Lisboa : Minist. da Educação. Inst. de investigação cientif. tropical. Centro de estudos de historia e cartografia antigua).

BRunot, F. (1966), Histoire de la langue française, t. III et IV, Paris, Colin.

Eyries, (1823), Notice « Pyrard», dans Biographie universelle, Paris, Michaud, 36, 348-50.

HaAse, A. (1935), Syntaxe française du XVII e siècle, nouv. éd. traduite et remaniée par M. OBERT, Paris, Delagrave.

MAT, M. (1979), Les influences françaises dans l'œuvre de l'abbé Duval-Pyrau, Études surle XVIII ${ }^{e}$ siècle, 6, 77-89.

PyRARD DE LA VAL, Fr. (1611), Discours du voyage des François aux Indes orientales, ensemble des divers accidens, adventures et dangers de l'auteur en plusieurs royaumes des Indes, et $d u$ sejour qu'il y a fait par dix ans, depuis l'an 1601. jusques en ceste année 1611, Paris, David Le Clerc.

- (1679), Voyage de F.P. de L. V. contenant sa navigation aux Indes orientales, Maldives, Moluques, et au Bresil : et les divers accidens qui luy sont arrivez en ce voyage pendant son sejour de dix ans dans ces païs. Avec une description exacte des mours, loix, façons de faire, police et gouvernement; du trafic et commerce qui s'y fait ; des animaux, arbres, fruits, et autres singularitez qui s'y rencontrent. Divisé en trois parties. Nouvelle edition, reveuë, corrigée et augmentée de divers traitez et relations curieuses. $<\ldots>$. Par le Sieur Du Val, geographe ordinaire du Roy, Paris, Louis Billaine. 
Sancier-Chateau, A. (1993), Introduction à la langue française du XVII siècle. 2. Syntaxe, Paris, Nathan.

Spillebout, G. (1985), Grammaire de la langue française du XVII siècle, Paris, Picard. 